\title{
A propósito del qué, cómo y para qué investigar en el campo de la justicia juvenil: la trastienda de un proceso de investigación en Rosario, Argentina
}

\author{
Karina De Bella \\ Doctora en Trabajo Social. Licenciada en Trabajo Social \\ Universidad Nacional de Rosario. Rosario, Argentina. \\ https:/ / orcid.org/0000-0002-4469-8143・ karina40debella@gmail.com
}

Resumen

Este artículo da cuenta de un proceso de investigación que se encuentra en curso en la ciudad de Rosario (Santa Fe, Argentina). El trabajo tiene como objeto mostrar dicho proceso que tiene la particularidad de ser llevado a cabo por un equipo de investigación conformado por docentes, investigadoras y licenciadas en Trabajo Social que no se encuentran vinculadas al ámbito académico, sino insertas profesionalmente en la administración de justicia. Analizar esta fructífera relación entre espacios académicos y espacios sociocupacionales, nos lleva a pensar en los dispositivos que se han desarrollado en la Universidad Nacional de Rosario y en las definiciones en cuanto al carácter de las investigaciones que desde allí se procura propiciar. La idea de trastienda es una invitación a conocer cómo se toman las decisiones en cuanto al qué, cómo y para qué investigar. La idea de proceso, y sobre todo en el contexto de la actual pandemia por COVID-19, nos lleva a visualizar los obstáculos y avances. Puntualizando en el campo de la justicia juvenil y partiendo de la necesariedad de la perspectiva sociojurídica, consideramos que el trabajo social contribuye a dicha perspectiva, como punto de encuentro para facilitar el diálogo entre diversos enfoques y áreas científicas (criminología, ciencia política, antropología, trabajo social). Específicamente, las presentes reflexiones surgen a partir del proyecto de investigación que se encuentra en curso, denominado "Justicia juvenil. Significados construidos por las y los jóvenes-adolescentes en relación con el proceso sociojurídico", apoyado en el enfoque metodológico cualitativo.

Palabras clave: Justicia juvenil; Investigación social; Trabajo Social; Enfoque sociojurídico.

Recibido: 27/08/2021 | Aprobado: 14/10/2021 | Publicado: 01/01/2022

(c) (1) Esta obra está bajo una Licencia Creative Commons Atribución-NoComercialCompartirIgual 4.0 Internacional.

Financiación o proveniencia del artículo: Artículo derivado del proyecto de Investigación y Desarrollo Justicia juvenil. Significados construidos por las y los jóvenes-adolescentes en relación con el proceso socio jurídico, (PID) 80120190100077UR desarrollado en la Universidad del Rosario, Argentina. Proyecto en curso.

¿Cómo citar este artículo? / How to quote this article?

Bella, K. de. (2022). A propósito del qué, cómo y para qué investigar en el campo de la justicia juvenil: la trastienda de un proceso de investigación en Rosario, Argentina. Prospectiva. Revista de Trabajo Social e intervención social, (33), 75-98. doi: 10.25100/prts.v0i33.11552. 
De Bella

\title{
About the What, How and Why of the investigation on the Field of Juvenile Justice: The Backroom of the Research Process in Rosario, Argentina
}

\begin{abstract}
This article gives an account of a research process that is underway in the city of Rosario (Santa Fe, Argentina). The work aims to show this process that has the particularity of being carried out by a research team made up of teachers, researchers and graduates in Social Work who are not linked to the academic field, but professionally inserted in the administration of justice. Analyzing this fruitful relationship between academic and socioemployment spheres leads us to think about the devices that have been developed at the Universidad del Rosario and the definitions in terms of the nature of the research that is promoted there. The idea of a backroom is an invitation to know how decisions are made as to what, how and why to investigate. The idea of process, and especially in the context of the current COVID-19 pandemic, leads us to visualize the obstacles and advances. Pointing out in the field of juvenile justice and starting from the need of the socio-legal perspective, we consider that social work contributes to this perspective, as a meeting point to facilitate dialogue between various approaches and scientific areas (criminology, political science, anthropology, social work). Specifically, these reflections arise from the ongoing research project called Juvenile Justice. Meanings Constructed by Young People-Adolescents in Relation to the Socio-legal Process, supported by the qualitative methodological approach.

Keywords: Juvenile Justice; Social investigation; Social work; Socio-legal approach.

Sumario: 1. Introducción, 2. Trabajo Social e investigación en el campo de la justicia juvenil, 3. Metodología, 4. Hallazgos, 4.1 Sobre cómo nos preparamos para investigar: la reflexividad en juego, 4.2. El diseño de la estrategia del trabajo empírico (momento anticipatorio), 4.3. El encuentro comunicativo con los sujetos investigados (momento dialógico), 4.4. Segundo y tercer momento de la reflexividad: el encuentro in situ y algunos hallazgos empíricos, 5. Conclusiones, 6 . Referencias bibliográficas.
\end{abstract}


De Bella

\section{Introducción}

El trabajo de investigación enunciado ${ }^{1}$ se desarrolla en el marco institucional del Centro de Investigación en Campos de Intervención del Trabajo Social (CIeCITS), dependiente de la Facultad de Ciencia Política y Relaciones Internacionales de la Universidad Nacional de Rosario (UNR). Dentro de los fundamentos de creación de dicho centro se enuncia la necesidad de vinculación del ámbito académico con los espacios socio profesionales del Trabajo Social (TS), posibilitando entonces la incorporación de trabajadores sociales insertas en dichos espacios socio profesionales y docentes. En este sentido, el CIeCITS se constituye como dispositivo institucional y concreto para llevar adelante la tarea investigativa. En dicho centro la tarea se organiza a partir de los distintos campos de intervención del TS (salud, género, educación, infancias/familias, etc.). Específicamente dentro del campo Infancias/familias, se creó la línea de investigación en Trabajo Social Forense. Compartimos con Alicia González Saibene ${ }^{2}$ que no se trata de establecer jerarquías sino de diferenciar, por medio de su caracterización, la investigación social de la intervención social del TS, recuperando en ese acto el sentido y la trascendencia de su articulación. De este posicionamiento resulta la comprensión de la necesidad del TS de producir conocimientos mediados por la lógica investigativa, con el fin de inscribirnos en las ciencias sociales y teniend o como horizonte los planteos de nuestra nueva Ley Federal de Trabajo Social (2014) ${ }^{3}$. En este contexto, formamos un equipo de trabajo con docentes investigadoras de TS y trabajadoras sociales con inserción profesional en el ámbito sociojurídico, específicamente en la administración de justicia provincial4. El desafío de hacer converger las tareas de investigación y de inserción profesional en el campo de la justicia es enorme, pero cuenta con fundamentos en la historia reciente, transitada en la provincia de Santa Fe, de construcción colectiva de un posicionamiento político y teórico ${ }^{5}$ ante los proyectos de reforma en la justicia juvenil. El mismo ubicó al TS como disciplina fundamental en la materia, para lo que contó con un apoyo fundamental por parte del Sindicato de Trabajadores Judiciales ${ }^{6}$ de nuestra provincia. Las integrantes del presente

1 Directora: Dra. Karina De Bella; co-directora: Dra. Daniela Polola; asesora: Dra. Elena Libia Achilli; auxiliares: Lic. María Eugenia Campbell, Lic. María de los Ángeles Caviglia, Lic. Emilce Mariel López, Lic. Isabel Fernanda Ojeda y Lic. María Ester Masferrer.

2 Nos referimos al documento emergente del proceso de formulación conceptual de la investigación "La cuestión de la investigación y la teoría en Trabajo Social" (PID 2012, 1POL184, UNR), presentado durante 2015 en diversos eventos nacionales -UBA, UNER- e internacionales -Universidad de Sinaloa, entre otroscomo propuesta para la creación de un centro de investigación.

3 Ley 27.072/2014, sancionada el 10/12/14. Sobre la investigación, ver capítulo III, art. 9, inc. 8, 9 y 10; y capítulo IV, art. 10.

4 Quisiera destacar que, con las trabajadoras sociales insertas en el poder judicial comparto dicho espacio socio ocupacional, además de la docencia e investigación universitarias. Esta particularidad, junto con mi rol de directora del proyecto, hizo posible canalizar las inquietudes en el CIeCITS y potenciar dicho centro.

5 La historia reciente de las acciones y propuestas realizadas por los trabajadores sociales del fuero de menores de la provincia de Santa Fe se encuentra ampliamente documentada en la ponencia presentada en el Congreso Nacional de Trabajo Social, realizado en agosto de 2018 en la ciudad de Santa Fe.

6 El Sindicato de Trabajadores Judiciales de la provincia de Santa Fe históricamente ha apoyado y ha representado a los trabajadores del trabajo social en las discusiones sobre justicia juvenil. 
De Bella

equipo de investigación participamos activamente del proceso reseñado. Insertas en el CIeCITS, a partir del registro de las intervenciones profesionales y las preocupaciones que nos fueron surgiendo, transformamos una serie de problemas empíricos en problemas de investigación y nos abrimos paso para consolidarnos en la lógica investigativa que dicho centro nos habilitó.

Así es que, durante el año 2018, las integrantes del equipo participamos en el seminario-taller de posgrado en el marco del doctorado en TS7 de la Facultad de Ciencia Política y RRII de la UNR denominado "Investigar procesos de Intervención Social”, a cargo de la Dra. Elena Achilli. De aquel programa académico nos interesa recuperar lo siguiente:

se propone generar un espacio de reflexividad que recupere tal experiencia disciplinar y la articule con los procesos de construcción de conocimientos que ella brinda. En tal sentido, se intenta focalizar en el quehacer de la investigación como camino que permita realizar tal articulación a partir del proceso que implica pasar del problema de intervención a la construcción de un problema de investigación. Se parte del supuesto de que todo proceso de investigación de una determinada problemática requiere llevar a cabo un conjunto de decisiones teóricas y metodológicas cuya dinámica e interrelación mutua ponen en juego determinadas lógicas de investigación "en acto" 8 .

Recuperamos el propósito del seminario-taller porque fue el espacio concreto donde el equipo pudo disponerse a diseñar el problema de investigación. Dicho diseño se materializó en la presentación del proyecto de investigación denominado “Justicia juvenil. Significados construidos por las y los jóvenes-adolescentes en relación con el proceso sociojurídico". Este trabajo fue aprobado y se revisaron algunos señalamientos indicados por la dictante de dicho curso. Resultando luego, en el año 2019, en la formulación de un Proyecto de Investigación y Desarrollo (PID). El proyecto fue aprobado en febrero de 2020 por evaluadores externos convocados por la UNR ${ }^{9}$. Transformado el proyecto inicial en PID, el equipo se amplía interdisciplinariamente con docentes antropólogas. Para el equipo de trabajo dicha aprobación significó un avance cualitativo para el desarrollo del proyecto. Dicho esto, es menester señalar que las condiciones de producción y progreso de la tarea investigativa se vieron afectadas por la irrupción de la pandemia de Covid-19, viéndose alterados los tiempos y la planificación diseñada ${ }^{10}$. En nuestro caso, insertas laboralmente

7 Cabe destacar que los egresados de la Facultad de Ciencia Política y RRII pueden acceder en forma gratuita a los cursos de doctorado.

8 Extraído del Programa Académico del Seminario-Taller, a cargo de la Dra. Achilli, dictado en el marco del Doctorado en Trabajo Social de la UNR, cursado entre los meses de junio y agosto del año 2018, con una carga horaria de $40 \mathrm{~h}$.

9 Las evaluadoras fueron las Dras. Claudia Krmpotic y Graciela Castro.

10 El gobierno nacional de nuestro país dispuso las medidas de aislamiento social preventivo y obligatorio, conocidas como ASPO (Aislamiento Social Preventivo y Obligatorio) mediante el Decreto 297/2020 de fecha 19/03/2020, publicado en el Boletín Oficial de la República Argentina el 20/03/2020, disponible en https://www.argentina.gob.ar/normativa/nacional/decreto-297-2020-335741/texto. De acuerdo con el acontecer de la situación sanitaria, dichas medidas se fueron prorrogando y modificando según las 
De Bella

en el poder judicial, nuestra tarea cotidiana presencial fue modificándose hacia la virtualidad, con períodos de asistencia de guardias mínimas, hasta el regreso al esquema de trabajo presencial con restricciones y bajo medidas de seguridad sanitaria.

El poder judicial, mediante resoluciones de la Corte Suprema de Justicia, hasta el día de hoy mantiene al personal con licencias y hay una clara indicación acerca de trabajar lo urgente y procurar restringir las citaciones para evitar la masiva concurrencia y circulación de las personas en las distintas sedes. La agenda diaria se vio modificada para respetar dichas indicaciones. En este marco, la dinámica de los procesos de intervención sufrió cambios, introduciéndose el uso de medios tecnológicos con mayor asiduidad, en pos de sostener el vínculo con las y los adolescentes infractores de la ley, sus familias, las instituciones y los programas, entre otros ${ }^{11}$.

No es menor señalar que nuestras propias realidades cotidianas se vieron alteradas durante el año 2020 y lo que va del 2021, las vivencias de temores y miedos frente a la enfermedad fueron una constante. La nueva realidad impuesta a partir de la irrupción de la pandemia generó cambios, ansiedades, angustias, las cuales de acuerdo con nuestros mundos internos y subjetividades propias nos instó a adaptarnos pasiva o activamente ${ }^{12}$ a la realidad. Es en este marco que presentamos la trastienda del proyecto y nos remitimos a las particularidades de nuestro proceso de investigación.

\section{Trabajo Social e investigación en el campo de la justicia juvenil}

Actualmente los juzgados de menores de la provincia de Santa Fe (Argentina) han sufrido modificaciones en cuanto a sus competencias debido al impacto de las normativas, específicamente la Ley 12.967/2009 (adecuación a la Ley 26.061/2005) en el Código Procesal de Menores -CPM- (Ley 11.452/1996), manteniéndose la Ley 22.278/1980. La competencia estrictamente penal se materializa entonces en el accionar de la secretaría penal. Asimismo, se mantiene la intervención profesional de los trabajadores sociales, que actúan previa derivación de dicha secretaría. Cabe destacar que es la política criminal la que define quiénes serán objeto del reproche penal ${ }^{13}$. Es en virtud de la competencia penal de los juzgados de

realidades provinciales. Particularmente en la provincia de Santa Fe, hubo, en general, consonancia con las disposiciones nacionales, variando solo en determinados momentos.

11 Un dato que nos resulta importante señalar, porque agudizó las imposibilidades de encuentro, fue que en la ciudad de Rosario hubo un paro del transporte público por 86 días. Este medio es el que utilizan mayoritariamente las y los jóvenes-adolescentes con quienes trabajamos.

12 Conceptos planteados desde la psicología social por Pichón-Rivière.

13 De acuerdo con la información proporcionada en el cuadernillo de material de estudio del curso destinado a los postulantes que pretenden ingresar como empleados administrativos al Poder Judicial de la Provincia de Santa Fe (2015), una conducta es reprochable, desde el punto de vista jurídico, cuando se infringe lo estipulado en las normativas vigentes de un orden social determinado (de acuerdo con el Código Penal Argentino). La política criminal es la que define en nuestro caso a qué edad y por qué tipo de causas se es punible. Si se determina entonces que la conducta fue llevada a cabo en forma voluntaria, tendremos que cotejar ahora que este encuadre perfectamente en una figura prevista en el Código Penal o en otra ley especial 
De Bella

menores, y por medio de este reproche, que las y los jóvenes-adolescentes ingresan al juzgado de menores, lo cual implica una judicialización del sujeto. Se define claramente que no puede haber otros motivos más que el reproche penal enmarcado en la definición de punibilidad: por edad y por tipo de delito según la Ley 22.278/1980, Régimen Penal de la Minoridad. Por vía de la Ley 26.061/2005 han desaparecido las categorías de abandono, riesgo, peligro moral o material, situación irregular o, las más modernas, de vulnerabilidad o disfunción familiar. En el actual esquema normativo provincial se encuentra la definición de funciones y competencias para el Trabajo Social. La intervención en este campo está directamente asociada al diseño, formulación, proposición, seguimiento y evaluación de las medidas de las que son objeto/sujeto de intervención las y los jóvenes-adolescentes imputados o declarados autores responsables.

En esta intervención profesional, que tiene como eje principal el proceso penal en curso, porque define la situación procesal del joven, se despliegan y organizan estrategias de trabajo. Para arribar a las mismas, el trabajador social recurre a las políticas públicas ofertadas por el Estado. Reúne el complejo legal normativo y la realidad social del joven. Aporta elementos para comprenderlo como sujeto teniendo en cuenta sus circunstancias personales -en las que atraviesa cambios subjetivos propios de la adolescencia-, así como la familia, la historia, la sociedad, el contexto, el territorio. Todo ello requiere de marcos conceptuales, reflexiones, definiciones técnico-operativas y propositivas, y el uso de instrumentos propios de la intervención profesional.

La tarea del trabajador social en este campo es de suma importancia por su proximidad con las y los jóvenes, por el conocimiento de su cotidianeidad y por la escucha entrenada que le permite crear un espacio propositivo y reflexivo de posible incidencia en la trayectoria de su vida. Se procura un proceso interventivo específico que opera a través de las medidas que el juez dispone. Lo que hace a la diferencia entre la justicia juvenil y la de mayores es que las y los jóvenes-adolescentes tienen derecho a que no se les aplique pena. Establece también la justicia juvenil que dentro de las condiciones que va a tener en cuenta el juez para evaluar la necesidad o no de la de aplicación de pena, una de ellas sea que se haya dispensado un "tratamiento" 14 tutelar. En este tratamiento aparece la cuestión de las medidas dispuestas. Por ello, si partimos desde este derecho que asiste al joven, la cuestión de las medidas cobra otro sentido, podríamos decir que las mismas pueden constituir un “ofrecimiento de posibilidades", 15 y es aquí donde radica, para nosotros, la importancia de

de naturaleza penal. Adecuada dicha conducta al tipo penal, es decir, a la figura que el Código describe como prohibida, la misma deberá además ser contraria a todo el ordenamiento jurídico. Este carácter se señala como el de la antijuridicidad de la conducta. Y, finalmente, el sujeto autor de la conducta típica y antijurídica deberá además ser "culpable", lo cual implica que dicho acto le deberá ser reprochable. Esto significa que al individuo le es exigible que hubiera comprendido que lo que hacía estaba prohibido, y que pudiendo haber elegido desarrollar la conducta adecuada, no lo hizo.

14 Este término fue abusivamente utilizado desde posiciones no compartidas por este estudio, de allí el uso de las comillas. No obstante, hay que tener presente que es la denominación en las leyes positivas.

15 Para ampliar sobre dicha conceptualización ver De Bella (2016). 
De Bella

las intervenciones del Trabajo Social: tornar posible algún desplazamiento, hacer alguna apertura de perspectiva, esbozar nuevos puntos de vista en un proceso con las y los jóvenes. Asumimos el concepto de "ofrecimiento de posibilidades" como perspectiva teórica orientadora del presente trabajo. De la ubicación que tiene el Trabajo Social en el proceso socio-jurídico descripto, interesa situarlo en su importancia, dada la dinámica diferenciada, sobre todo en la relación con los actores de dicho proceso, en tanto es muy disímil con otras experiencias. Tal es el caso en la provincia de Santiago del Estero donde, siguiendo a Medina (2021), la creación de la Oficina de Medidas Alternativas y Sustitutivas del Poder Judicial actúa en el control y supervisión de dichas medidas, impuestas por los juzgados de garantías, en causas judiciales que involucran tanto a jóvenes como adultos. Advertimos, de alguna manera, la pérdida de la especialidad en el abordaje en tanto se reduciría la intervención profesional sólo a la fase ejecutiva y dicho accionar tanto para adultos como para jóvenes, podría obturar la necesidad de una respuesta estatal diferenciada en justicia juvenil resultando diluida y poco específica.

La indagación en el surgimiento del entramado jurídico de intervención socio penal sobre niños y adolescentes ha conllevado una profusa producción bibliográfica a nivel internacional, en particular a partir de la segunda mitad del siglo $X X$, especialmente en Europa (De Leo, 1985; Donzelot, 2008) y Estados Unidos (Platt, 2006). También a nivel latinoamericano se registran antecedentes de investigación que indagan en diversos aspectos del sistema de justicia penal juvenil: Beloff (2001); Beloff, Freedman y Terragni (2013); Castrillón-Valderrutén (2005); Cillero-Bruñol (1998); D’ Antonio (2004); Dassi \& Reis (2008); De Andrade-Castro (2005); Díaz de León y González-Placencia (2004) y GarcíaMéndez (1994, 1997, 2008). En el campo de las ciencias sociales en Argentina, por su parte, podemos mencionar los abordajes desde el campo socio antropológico de la estructura jurídico-burocrática en Buenos Aires: Graziano (2017); Grinberg (2004); Guemureman (2011, 2020); Roovers (2003); Villalta (2004, 2013, 2021); y trabajos de análisis del funcionamiento interno de institutos de privación de la libertad (Daroqui, López y Cipriano-García, 2012; Miguez, 2008; Miguez y González, 2003; Nebra 2015; Tedesco, 2007).

En la provincia de Santa Fe existen producciones que abordan desde la perspectiva jurídica el entramado tutelar a nivel histórico, así como investigaciones desde disciplinas como la psicología (Degano, 2004) y, desde el campo antropológico, investigaciones sobre vulneraciones de los derechos humanos de personas menores de edad privadas de la libertad (Ruiz-Bry, 2011), como el análisis del entramado burocrático de intervención tanto a nivel civil como penal en la ciudad de Rosario (Polola, 2004).

Advertimos al respecto que, en muchas ocasiones, los análisis se inclinan en demasía por los aspectos procesales o bien realizan un abordaje unilateral del campo y solo toman en cuenta la situación de privación de la libertad. Sin embargo, recientemente Villalta (2021) realiza un recorrido donde señala contribuciones de investigaciones desde la antropología 
De Bella

política y jurídica, del campo de la etnografía de la niñez, de la antropología de la educación, del campo de los estudios sociales de infancia y juventud, etc., que abonan a la constitución del campo institucional, donde se inscribe la presente investigación. Aportes que, estimamos contribuirán a la complejización de este trabajo en su desarrollo y argumentación ${ }^{16}$.

El TS interviene con los sujetos judicializados en un amplio abanico de situaciones, en las que la privación de libertad puede ser una de ellas, entre otras. También queremos señalar que dichos análisis describen mecanismos de control social del Estado, brindan una historización acerca de la niñez, aportan a la identificación de modos de intervención, pero no son análisis de disciplinas con la distintiva marca del TS en relación con la intervención profesional directa. Por ello, nos parece sumamente importante analizar las contribuciones existentes del TS en materia de justicia juvenil. Contamos con los aportes específicos sobre las incidencias profesionales del TS en esta área (De Bella, 2016; Velurtas, 2017); estudios acerca de la historia profesional en justicia juvenil y sobre los procesos sociojurídicos en relación con las políticas públicas y programas específicos como el de libertad asistida; reflexiones en torno de la pretendida responsabilidad penal juvenil (Marcón, 2011, 2013, 2017), entre otros. No obstante, se verifica un área de vacancia en investigaciones desde Trabajo Social que aborden los procesos sociojurídicos integralmente.

En este sentido, el problema de investigación se formula desde una perspectiva del TS en su carácter integral, interdisciplinario e intersectorial, conjugando los campos social y jurídico. Uno de los núcleos de nuestras preocupaciones lo constituyen los sujetos de la intervención en procesos sociojurídicos. Sujetos que situamos en el transitar de la adolescencia. Stella Maris Firpo (2013) plantea que entonces "se transita una zona de frontera plena de conflictos y a su vez de múltiples potencialidades, en la que los adolescentes necesitan de otro que acompañe, legitime, habilite y los ayude a ingresar en las responsabilidades" (p. 27). Es por ello que en el presente trabajo hemos definido hablar no de jóvenes, sino de jóvenes-adolescentes.

\section{Metodología}

Nuestro ejercicio profesional nos sitúa en una relación diaria con las y los jóvenesadolescentes que se encuentran transitando la adolescencia y que han infringido las leyes penales. En ese cotidiano profesional aparece el cotidiano de esos sujetos, en el que expresan sus situaciones familiares, vinculares, grupales, afectivas, escolares, sanitarias, habitacionales, así como sus hábitos de consumo. En este sentido coincidimos con Achilli (2015): “cuando hablamos de 'lo cotidiano' consideramos que en cualquier campo de la vida social se configuran un conjunto de prácticas, relaciones, significaciones diversas y

16 Dado que el presente trabajo, es sobre una investigación que se encuentra en curso, los insumos que señala Villalta se tomarán al momento de analizar lo producido en el trabajo de campo. 
De Bella

heterogéneas que construyen sujetos particulares al interior de una realidad concreta" (p. 22).

Dadas las posiciones teóricas enunciadas, decidimos asumir el planteo del enfoque socio antropológico desarrollado por esta autora: "consideramos la importancia de analizar las relaciones y procesos cotidianos no a modo de formulaciones vacías que silencian a los propios protagonistas sino reconociendo el conjunto de representaciones, significaciones y sentidos que generan los sujetos como parte de un conjunto social" (p. 25).

Teniendo en cuenta los antecedentes y las consideraciones teóricas formuladas, entendemos que el problema de investigación se centra en la parcial vacancia de conocimientos que tengan como eje la voz de los protagonistas, en este caso las y los jóvenesadolescentes que se encuentran transitando el proceso judicial. En especial, la vacancia se advierte sobre el conjunto de significaciones y sentidos que estas y estos jóvenesadolescentes atribuyen a dicho tránsito en el que se dan las intervenciones profesionales del Trabajo Social. Aquí se encuentra el cruce que denominamos enfoque sociojurídico ${ }^{17}$. Se trata, ahora, de investigar cómo los sujetos de la intervención significan de modos diversos el tránsito por el proceso judicial. Esta particularidad imprime un carácter distintivo a la acción penal porque el sujeto es abordado desde la lógica del proceso judicial y desde la lógica del sujeto sociohistórico. En este sentido, nos formulamos las preguntas siguientes: ¿el transitar estas dos lógicas produce dicotomías en las/los jóvenes-adolescentes respecto de su subjetividad?, ¿cómo significan las/los jóvenes-adolescentes estas lógicas a partir de las intervenciones profesionales del TS?, ¿cómo han repercutido en sus vidas cotidianas las medidas dispuestas judicialmente? En este sentido, nos propusimos la siguiente hipótesis: las y los jóvenes-adolescentes que han infringido las leyes penales significan de modos diversos el proceso judicial en relación con las intervenciones profesionales del Trabajo Social en sus vidas cotidianas. El objetivo general se definió por conocer de qué modos significan las y los jóvenes-adolescentes que han infringido leyes penales dicho proceso.

Para desarrollar el presente trabajo optamos por un abordaje metodológico cualitativo desde el enfoque socio antropológico referido. Realizaremos una serie de entrevistas semiestructuradas en profundidad a las y los jóvenes-adolescentes, ya que entendemos que

este tipo de técnica cualitativa sostiene reiterados encuentros cara a cara entre el investigador/a y el/la los/as informantes/es, encuentros dirigidos a la comprensión de las perspectivas que tienen estos respecto de sus vidas, experiencias o situaciones, tal como las expresan con sus propias palabras. (Taylor y Bodgan, 1998, p. 101)

17 Esta conceptualización se desarrollará más adelante. 
De Bella

Se trabajará con sentencias judiciales, las que asumimos en términos de documentos. Como señala Valles (citado en Fernández, 2013), "los documentos son producciones culturales que condensan posiciones, intereses, valores, expectativas de quienes las formulan y sobre quienes se pretende incidir" (p. 58). En este sentido, es relevante situar los documentos siguiendo a Muzzopappa y Villalta (2011) en el campo de lo estatal como construcción analítica y que posibilita abordar dichos documentos producidos por el Estado como resultado de relaciones de poder que lo constituyen y atraviesan. Asumiremos las sentencias en el sentido que señala De Bella (2016), "como reconstrucciones de procesos sociales biográficos" (p. 267). Asimismo, resultará relevante el análisis de Guemureman (2020) respecto de sentencias judiciales donde muestra el peso de los juicios morales en la ponderación de las penas que analiza. Si bien es menester plantear que, en la provincia de Santa Fe predominan las sentencias absolutorias por innecesaridad de aplicación de pena ${ }^{18}$.

En cuanto al recorte empírico, trabajamos, por una parte, con jóvenes-adolescentes entre 16 y 18 años, en proceso judicial, con al menos un año de inicio de la intervención del TS (a fin de que pueda observarse el despliegue de las medidas judiciales); y, por otra parte, con jóvenes-adolescentes de 18 años en adelante (declarados autores responsables). El recorte temporal se define por quienes en el proceso judicial cuentan con la segunda sentencia resuelta entre 2019 y 2021.

\section{Hallazgos}

\subsection{Sobre cómo nos preparamos para investigar: la reflexividad en juego}

Leticia Muñiz-Terra, Juliana Frassa y María de la Paz Bidauri (2018) plantean que, para el investigador, la reflexividad, si bien se inicia cuando este delimita su problema de investigación, adquiere características distintivas durante las diferentes etapas del trabajo de campo: en el diseño de la estrategia del trabajo empírico (momento anticipatorio), en el encuentro comunicativo con los sujetos investigados (momento dialógico) y en una primera revisión de la entrevista realizada (momento analítico).

Teniendo en cuenta los tres momentos enunciados, quisiéramos referirnos a cuestiones básicas, no siempre explicitadas en relación con la conformación del equipo de investigación, los roles, las funciones a desplegar y la tarea de investigar. Este es un punto central para avanzar en el desarrollo de un proyecto. Una de las cuestiones importantes y recomendables es procurar estar en tarea. La noción de tarea tiene relevancia para pensar al grupo dotado de una operatividad en función en un doble aspecto: explícito e implícito. Por explícito, nos referimos a la definición de la problemática a investigar, la formulación de la

18 Este dato no es menor y refuerza la perspectiva teórica desarrollada en términos de "ofrecimiento de posibilidades" como resignificación de la idea de "tratamiento tutelar". Señalo la importancia, en este punto, de poder reconocer la variabilidad en las decisiones socio-jurídicas de acuerdo con contextos institucionales y políticos particulares. 
De Bella

hipótesis y los objetivos. Lo implícito, por su parte, está vinculado al conocimiento que de la problemática poseen las integrantes del equipo de investigación. Esta dimensión ha puesto en tensión nuestras concepciones previas, prejuicios, posicionamientos y temores en relación a ser parte del propio proceso judicial descripto, a la vez que buscar adquirir una distancia óptima que nos permitiera discriminar entre la propia inserción socio ocupacional, el quehacer cotidiano profesional y la construcción del rol de investigadoras. Nueva y doble pertenencia dentro del campo que necesariamente debía asumirse una manera crítica.

\subsection{El diseño de la estrategia del trabajo empírico (momento anticipatorio)}

Una de las cuestiones que nos permitió desarrollar el rol investigadoras se vinculó a las lecturas referentes a la categoría de reflexividad. Si bien la bibliografía consultada se remite a antropólogos y sociólogos, nos resulta pertinente para el trabajo social. De dichas lecturas, diversos autores nos proporcionaron pistas concretas para tener en cuenta en el proceso de investigación. En este sentido, Guber (2018) señala que en los últimos veinte años la popularidad de la etnografía y la reflexividad se concentró en cierta perspectiva sobre la metodología, limitándose a autorizar la inclusión de la persona del investigador en la aplicación de una técnica -entrevista en profundidad y observación participante-. Ciertos aspectos de la persona del investigador modelan el campo de trabajo y por lo tanto la investigación. A Guber le interesa llegar al punto de "cómo la reflexividad no solo debe ser analizada para comprender la producción subjetivada (del investigador) de los datos de campo, sino que debe ser incorporada para articular y comprender las distintas fases de la investigación" (p. 52). En este sentido, la autora retoma lo que el antropólogo estadounidense Clifford Geertz advertía junto a Heinz Kohut acerca de la distinción entre "conceptos de experiencia cercana" y de "experiencia distante".

Un concepto de experiencia cercana es, básicamente, el que alguien, un paciente -un sujeto, en nuestro caso un informante- podría él mismo y sin esfuerzo usar para definir lo que él o sus compañeros ven o sienten, piensan, imaginan, y que él podría entender cuando se aplique de modo similar a los demás. Un concepto de experiencia distante es aquel que los especialistas de un tipo u otro -un analista, un experimentador, un etnógrafo, incluso un cura o un ideólogousan para avanzar hacia sus objetivos científicos, filosóficos o prácticos (1983, p. 57; mi traducción). (Geertz citado en Guber, 2018, p. 54)

Los conceptos de experiencia cercana nos dan claves fundamentales para comprender, elaborar y hasta discutir nociones teóricas abstractas. Asimismo, Gustavo Lins-Ribeiro (1989) plantea que,

la constante fluidez teórica y metodológica entre las distintas disciplinas que analizan la experiencia humana, lleva a los antropólogos a buscar diferentes especificidades de la perspectiva de la antropología. Este debate trae a un primer plano la discusión sobre la relación individuo/sociedad, estableciéndose en las ciencias sociales, en términos de tensión existente entre los enfoques subjetivistas y objetivistas. Propone que la salida solo puede plantearse en 
términos de una comprensión dialéctica [...] En esta dialéctica de aproximación/distanciamiento está embutida una paradoja central de la experiencia existencial de la práctica del antropólogo y definida con la siguiente expresión you can never go native: uno nunca se transforma en nativo. El descotidianizar parecería ser, no solo una manera de ser, sino de vivir en una búsqueda de solucionar la tensión aproximación/distanciamiento para revelar a través de una experiencia totalizante los elementos constitutivos de la realidad social. (p. 67)

Al respecto, las nociones de "experiencia distante" y de "descotidianizar" nos permitieron, al avanzar en el proyecto, cuando sentíamos que tal vez estábamos perdiendo el eje o agregando cuestiones que eran para otras investigaciones, volver a centrarnos en los objetivos de nuestro proyecto. A su vez, esta perspectiva dialéctica entre aproximación y distanciamiento nos posibilitó estar atentas al momento del diseño de las entrevistas, a nuestras propias naturalizaciones, sobre todo, al uso del lenguaje jurídico. Las y los autores citados nos proporcionaron importantes elementos de reflexión, específicamente en nuestra doble inserción, como trabajadoras sociales en la administración de justicia y como investigadoras en el propio campo. En este sentido, agregamos siguiendo a Muñiz-Terra, Frassa y Bidauri (2018) que "las reflexiones metodológicas recientes realizadas tanto desde el campo de la antropología como desde la sociología han puesto de relieve la centralidad del encuentro entre el investigador y los sujetos investigados en la instancia del trabajo de campo" (p. 120). Las autoras asumen, a partir de los desarrollos de Giddens, que los actores sociales son seres reflexivos, es decir que poseen capacidades para comprender su experiencia y la de los otros actores. Giddens distingue, por su parte, la existencia de dos aspectos en la reflexividad: la del observador social y la de los actores cuya conducta él pretende analizar. La reflexividad del investigador/observador social consiste en ser consciente de su posición en el espacio social, adoptando, a lo largo de su investigación, una mirada crítica que cuestione su mundo de representaciones para poder aprehender y comprender las particularidades de las biografías que pretende analizar. En este sentido, "las explicaciones de las acciones comunes que realiza el investigador son en sí mismas una realización práctica monitoreada reflexivamente" (Giddens, 1977 citado en Muñiz-Terra, Frassa y Bidauri, 2018, p. 121). Entendida como una estrategia de "vigilancia epistemológica", la reflexividad del investigador debería expresarse, según Bourdieu (1997, citado en Muñiz-Terra, Frassa y Bidauri, 2018), en tres niveles diferenciados: “la reflexividad sobre los condicionamientos que devienen de la posición ocupada por el investigador en el espacio social, la reflexividad sobre la posición que ocupa el sociólogo dentro de las propias ciencias sociales, y la reflexividad en relación a la potencial confusión entre los puntos de vista del sociólogo (teoría) y la realidad social (práctica)” (p. 121).

$\mathrm{Al}$ respecto de la estrategia de vigilancia epistemológica, reflexionamos sobre nuestra construcción del rol de investigadoras en la posición que ocupamos como trabajadoras sociales en el poder judicial y sus implicancias en las representaciones del sujeto que participaría en las entrevistas. Nos surgieron interrogantes acerca de si esta pertenencia 
De Bella

institucional condicionaría las respuestas de los entrevistados, si los haría sentirse coaccionados frente al escenario y espacio judicial.

En cuanto a nuestra posición disciplinar en relación con el derecho, analizamos la perspectiva socio jurídica para comprender nuestro propio proceso en el espacio del poder judicial, donde predomina el saber jurídico. En este sentido y con los aportes de CalvoGarcía (2010), nos situamos desde dicha perspectiva, que pretende integrar la teoría del derecho con la perspectiva de las ciencias sociales en el ámbito de las ideas jurídicas para abrir espacios y desarrollar una teoría socio jurídica del derecho que se nutra de los análisis e investigaciones sociales. Esta perspectiva pretende facilitar la comprensión de los fenómenos jurídicos en sentido amplio y plural, aunar herramientas metodológicas de los dos campos, incorporar investigaciones de la teoría social (no reducibles a la sociología) con apoyatura en otras ciencias sociales orientadas a la investigación. El autor, al plantear la idea de teoría socio jurídica, entiende a la misma como punto de encuentro para facilitar el diálogo entre perspectivas y áreas científicas diversas: criminología, ciencia política, antropología. Nosotras agregaríamos, por qué no, al Trabajo social.

La perspectiva socio jurídica nos fortaleció para establecer y fundamentar el diálogo con las autoridades judiciales, justificar la utilidad del enfoque para el mejoramiento de las prácticas judiciales en su conjunto y, específicamente, para obtener las autorizaciones para la realización de las entrevistas y el uso de documentos institucionales. Cabe destacar que este proceso de habilitación fue consensuado por uno de los jueces ${ }^{19}$ de la justicia juvenil de nuestra provincia, bajo los principios de confidencialidad de los datos y ética de la investigación de acuerdo con las normativas vigentes en la materia. Este aspecto, tan fundamental, cabe ser señalado en tanto muestra movimientos de apertura de los campos social y jurídico, y su necesaria vinculación. Al respecto Lacey (citado en Calvo-García, 2010) es enfático y sostiene que "el aislamiento de la teoría del derecho de la teoría social y los estudios sociojurídicos es un error" (p. 387). Podríamos decir, que este movimiento se relaciona con las teorías críticas del derecho positivista.

Otra reflexión que nos parece pertinente respecto del Trabajo Social y la presente experiencia de investigación es que nos permite resituarnos en el espacio socio ocupacional desde las propias incumbencias profesionales definidas por la Ley Federal de Trabajo Social (2014) sancionada el 10/12/14, y, sobre todo, nos permite salirnos del histórico lugar de la "auxiliaridad" en el campo de la justicia -si bien es extensible a otros campos-, para desarrollar un lugar disciplinar distinto, desde la colaboración e igualdad entre las disciplinas en el campo de las ciencias sociales.

19 Juez de primera instancia de distrito, Dr. Alejandro Cardinale. 
De Bella

\title{
4.3 El encuentro comunicativo con los sujetos investigados (momento dialógico)
}

En relación con el tipo de entrevista definida para el proyecto, nos decidimos por la entrevista semiestructurada, considerando y continuando el planteo de Muñiz-Terra, Frassa y Bidauri (2018), según el cual:

\begin{abstract}
la entrevista biográfica puede adquirir distintas características de acuerdo con el tipo de instrumento que se utilice. Así, mientras algunas investigaciones realizan entrevistas biográficas semiestructuradas, otros estudios desarrollan entrevistas biográfico-narrativas. Así, las entrevistas biográficas semiestructuradas, que han sido también llamadas entrevista en profundidad (Valles, 1997; Ruíz Olabuénaga, 2012), y entrevista etnográfica de historia de vida (Sautú, 1998) se sustentan en una guía de preguntas confeccionada por el investigador en función de sus supuestos teóricos previos y en sus prenociones en relación a la historia de vida del actor social. (p. 123)
\end{abstract}

Es necesario recordar que es siempre el propio investigador quien debe decidir metodológicamente qué tipo de entrevista utilizar, pudiendo a su vez combinar diferentes tipos en distintos momentos de una misma interlocución. La aplicación de una u otra entrevista se vincula en primer lugar con la manera en que se pretende construir conocimiento. Si bien desde la perspectiva biográfica se supone que todo ser humano posee capacidad de reflexión, creemos que, en el marco de una entrevista biográfica, quien narra despliega una reflexión especial sobre su propia vida ya que, en palabras de Bertaux (1999), "el sujeto no recita su vida, sino que reflexiona sobre ella mientras la cuenta" (p. 10). En segundo lugar, resulta también importante señalar que el tipo de entrevista que se adopte se vincula con las posibilidades y limitaciones que presenta el trabajo de campo biográfico. Por otro lado, una particularidad del trabajo con entrevistas biográficas es la posibilidad de realizar una segunda y tercera entrevista con el mismo narrador para comprender en profundidad procesos o situaciones que fueron solo mencionadas en el primer encuentro comunicativo y que el entrevistador considera relevante recuperar. Ahora bien, más allá de estas características, resulta significativo mencionar que la entrevista biográfica, con sus distintos instrumentos, es una invitación que se realiza al actor social a pensar y narrar su vida. Lo interesante radica aquí en recuperar

cómo el entrevistado desarrolla su reflexividad, repensando y narrando su propia vida, otorgándole un sentido y planteando relaciones causales y temporales entre los distintos eventos evocados y relatados (Piña, 1986, p. 25), en el marco de la investigación en la que se encuentra participando. (Muñiz-Terra, Frassa y Bidauri, 2018, p. 125)

En este sentido, la categoría de reflexividad se fue internalizando en las integrantes del equipo como resorte constante del proceso en la tarea investigativa. Dicha reflexividad nos permitió analizar cómo llevar adelante las entrevistas semiestructuradas definidas en el proyecto. Discutimos que sería más apropiado definir quién debía ser la entrevistadora, si 
De Bella

la trabajadora social que acompañó al sujeto en el proceso sociojurídico desde el inicio hasta su finalización, u otra trabajadora social parte del equipo de investigación que no hubiera participado en dicho acompañamiento. En ambos casos con un miembro del equipo en función de observación. En un primer momento analizamos los pros y contras de cada una de las opciones. Las primeras experiencias de prueba del instrumento (realización de las entrevistas semiestructuradas) se realizaron en el marco de la primera opción, destacándose como pros y contras los siguientes: a su favor la existencia de un vínculo de confianza y conocimiento entre la entrevistadora y el sujeto entrevistado $\mathrm{y}$, tal vez, como contra, que dicha confianza podía obturar la apertura del sujeto para plantear cuestiones más de índole crítica, entre otras. Estas apreciaciones están en proceso de poder ser contrastadas mediante la prueba de intercambio con la entrevistadora que no realizó el acompañamiento y que se encuentra en el rol de observadora con quien sí lo realizó. La puesta en práctica, finalmente, nos permitió evaluar que lo más pertinente es que la entrevistadora sea quien lleve adelante el acompañamiento en el proceso judicial, siendo disipadas las dudas en cuanto a que el entrevistado se viera limitado en sus expresiones. Las y los jóvenes-adolescentes manifiestan sus sentires y pensares sin obstáculos. Podríamos decir que el vínculo establecido durante el proceso judicial con la trabajadora social es habilitante para la circulación de la palabra. El rol de la observadora asimismo se volvió central a la hora de contextualizar el momento de la entrevista. Para ello, definimos qué aspectos serían válidos de ser observados y construimos indicadores a tal fin. Una de las cuestiones que nos surgió fue la de analizar la comunicación: si se comprendían las preguntas propuestas o había que revisar el guion, cómo era el clima que se producía durante la misma, si había silencios, gestos, etc.

En cuanto a la conformación de los subgrupos dentro del equipo de investigación, para realizar esta tarea fue muy importante establecer los roles y funciones, para integrarlos y que no se generara suplementación. Asimismo, acordamos que, si la observadora advertía la necesidad de una repregunta, una instancia de esclarecimiento o la pertinencia de una intervención, se habilitaría el espacio para su formulación. De esta forma integrada se potenció el momento de las entrevistas.

Retomamos también de Muñiz-Terra, Frassa y Bidauri (2018) la idea de poner en juego una reflexividad de tipo preliminar, decisoria y anticipatoria del trabajo empírico al momento de definir a quiénes se va a entrevistar, cómo se presentará y realizará la invitación, y en dónde y de qué manera se desarrollará la interacción. El asunto de a quiénes entrevistar fue definido según nuestro referente empírico: las y los jóvenes-adolescentes que cuentan con las dos sentencias judiciales y se encuentran en la finalización del proceso sociojurídico. La invitación se produce luego de la instancia donde nosotras notificamos la sentencia judicial, para ello se cursa una citación formal a la sede del juzgado interviniente. Este punto ilustra el acuerdo institucional logrado y el consenso con el juez de habilitar el espacio para desarrollar la investigación. 
De Bella

Luego de la lectura de la sentencia, se realiza la invitación para participar en la investigación. En este punto, la experiencia que hemos tenido es que se trata de un momento de cierre para las y los jóvenes-adolescentes, que produce una carga de emociones importante. La sentencia, como reconstrucción biográfica, crea y recrea un situar de los sujetos en clave de recuperación histórica de la experiencia vivida a partir del ingreso al sistema penal. Por lo que es un momento muy movilizante y de reencuentro con un pasado reciente. De allí radica, a su vez, la importancia de incluir la tarea de la observación. Es en esta circunstancia que se presenta el proyecto de investigación y se invita al/a la joven a participar, informándole sobre el consentimiento necesario y la discrecionalidad en el tratamiento de la información.

Otra de las cuestiones que nos surgió y nos hizo reflexionar sobre el tipo de entrevista a realizar y el momento para la misma fue que, como se trata de una instancia de cierre del proceso sociojurídico, formalmente cesa allí la posibilidad, desde ese marco, para un nuevo encuentro. Sin embargo, nos encontramos con que todos los sujetos entrevistados, ante la propuesta de un nuevo encuentro, nos manifestaron su disposición a participar. Todo este primer momento significa poner en juego una reflexividad de tipo preliminar, decisoria y anticipatoria al trabajo empírico.

Dado que nuestro proyecto de investigación está en curso y nos encontramos realizando las entrevistas, podríamos decir que estamos transitando parte del segundo y tercer momento de la reflexividad. Siguiendo a Bourdieu y Wacquant (2005, citados en Muñiz-Terra, Frassa y Bidauri, 2018), el segundo momento para el investigador es cuando se produce el encuentro in situ. En esa relación comunicativa que se establece en una entrevista biográfica, el investigador puede y debe dejarse sorprender por la narración, intentando poner en suspenso tanto su sentido común como sus construcciones teóricas previas en relación con la historia relatada. Esa disposición receptiva de quien escucha abre las puertas al mundo de representaciones del otro y permite comprender en profundidad el relato. Una vez que ha concluido el encuentro narrativo, el investigador se enfrenta al tercer momento de reflexividad, durante el cual debe revisitar sus preguntas iniciales, analizar los conocimientos construidos, vincular ese material con información proveniente de otras fuentes, ya sean primarias o secundarias, y decidir si resulta necesario acceder a un nuevo encuentro para pedir ampliaciones y conversar con el "otro" acerca de su interpretación científica. 
De Bella

\subsection{Segundo y tercer momento de la reflexividad: el encuentro in situ y algunos hallazgos empíricos}

Tal como se desarrolló en la introducción, la coyuntura actual en el marco de la pandemia incidió en la planificación del proyecto, sobre todo en la posibilidad de realizar las entrevistas en su totalidad, debido a las medidas sanitarias que modificaron nuestro quehacer profesional. En este sentido, las restricciones de cantidad de personas que pudieran transitar en la sede del tribunal modificaron el esquema temporal definido. No obstante, pudimos hacer la prueba del guion de la entrevista semiestructurada, advertir las necesarias modificaciones y continuar con el nuevo guion.

Nos resulta importante compartir que el guion inicial se encontraba cargado de nuestras apreciaciones teóricas, formulado en un lenguaje, en algunos casos, dificultoso de ser comprendido por los entrevistados. Advertimos entonces nuestras propias naturalizaciones discursivas expresadas en categorías jurídicas de uso frecuente en nuestro quehacer profesional. De allí que la idea de descotidianizar, que nos trae Lins-Ribeiro (1989), nos resultara útil. Asimismo, surgió la necesidad de abrir más el guion, en el sentido de incorporar el sentir de las y los jóvenes-adolescentes. Rectificado y probado nuevamente, logramos adecuar el instrumento metodológico.

Respecto de algunos hallazgos encontrados durante el proceso, podemos referir lo siguiente:

- Efectivamente, el momento del encuentro y la notificación de la sentencia genera la movilización de diversas emociones. La recuperación del pasado reciente y la idea de las sentencias como construcciones biográficas sitúan, en ese primer momento, a las y los jóvenes-adolescentes en parte de su propia historia, singular y en un determinado contexto sociohistórico. El equipo de investigación tiene que respetar los tiempos que necesita el entrevistado, hacer lugar a sus silencios, esperar y calibrar el segundo momento para iniciar lo concerniente a la investigación en sí. Este momento implica para los entrevistadores asumir las propias ansiedades y situarse en el rol de investigador.

- Todas y todos los entrevistados manifestaron su consentimiento a participar, y podríamos decir que observamos predominó el asombro y la alegría al ser valorados como sujetos protagonistas del proyecto de investigación.

- Respecto del primer momento de contacto con el "tribunal" e inicio del proceso judicial, son coincidentes las respuestas de las y los jóvenes-adolescentes que señalan sentir que no comprendían muy bien de qué se trataba, sobre todo en cuanto a la interpretación de frases tales como "declaración indagatoria". Miedo, temor, confusión son los primeros sentires. El entendimiento y el esclarecimiento de esa instancia, así como la comprensión de en qué consistía el proceso judicial 
De Bella

les llevó un tiempo de acomodamiento, encontrando en la defensa técnica y en el encuentro con el espacio de Trabajo Social, sitios de disipación de dudas, aclaraciones, etc. En este sentido, los entrevistados nos advierten sobre el uso del lenguaje jurídico y la vulneración de su derecho a la comunicación como aristas a profundizar y problematizar con todos los actores institucionales del proceso sociojurídico en curso.

- La mayoría de las y los entrevistados refiere que estar físicamente en el escenario judicial, sea que se hayan presentado vía citación de un juez o trasladados por fuerzas de seguridad -policías o agentes penitenciarios- al encontrarse privados de la libertad, les produjo miedo, vergüenza, no saber muy bien qué les iba a pasar luego, angustia. Al respecto, nos resulta pertinente la relación con el concepto de crisis, en su doble aspecto de caos y oportunidad.

- Aparece en las y los entrevistados una valoración positiva del encuentro con las trabajadoras sociales, en frases tales como: "me gustaba venir hablar con vos", "me sentía bien, cómoda/o", "me ayudaron mucho", "sentía que podía hablar de cosas que me pasaban", "me sentía escuchada/o". La mayoría no había tenido un contacto con trabajadores sociales en otras instituciones, muchas/os de las y los entrevistados refirieron que era la primera vez que hablaban acerca de lo que sentían y les sucedía. Dicha valoración nos remite a la idea de proceso y construcción de un vínculo habilitante y afectivo que posibilita la puesta en acto de una escucha entrenada por parte de las profesionales del Trabajo Social. En este sentido, de los antecedentes citados, nos interesa trabajar próximamente, como parte del desarrollo de la presente investigación, en relación con la producción de María Florencia Graziano (2017), quien aborda en su libro "Pequeños Juicios" el rol de la observación tutelar. Si bien hay que destacar, que, en la presente investigación no se aborda las prácticas de las trabajadoras sociales en sí mismas, sino en la significación que las y los entrevistados le asignan. Asimismo, los contenidos que aporta la autora en relación con las estrategias moralizantes de la administración de justicia penal, serán un recurso valioso para distinguir diferencias, matices o similitudes entre estudios que se vinculan con la administración de justicia para los jóvenes-adolescentes en juzgados nacionales penales de menores de la ciudad de Buenos Aires y los juzgados de menores de la provincia de Santa Fe. Al respecto, no es menor señalar que los arreglos institucionales y las prácticas en la administración de justicia, aún bajo un marco normativo común difieren en cada provincia. Por lo cual, la contextualización es sumamente relevante.

- La mayoría de las y los entrevistados, en la recuperación de su historia reciente, hacen referencia al acompañamiento familiar, la presencia de algún adulto responsable, en algunos casos sus madres, en menor medida sus padres y/o suegros, lo que nos ratifica la pertinencia del concepto de adolescencia que hemos introducido. 
- Las y los entrevistados significan la irrupción del conflicto penal como un límite, podríamos enunciar, al encontrarnos con decires tales como: "si no me pasaba esto, terminaba presa/o o muerta/o", "sentí vergüenza, acompañaba a mi hermano al tribunal y ahora estoy yo". Nuevamente la idea de crisis en su doble aspecto y la idea de límite, no como castración sino como instancia habilitante, nos proporciona indicios para profundizar en el momento propio de la adolescencia como uno de tránsito, zona de frontera y necesidad de otro que acompañe. Asimismo, trágicamente, nos alerta sobre las condiciones concretas de existencia de estos adolescentes, donde las posibles proyecciones introyectadas están vinculadas a la privación de la libertad o la muerte. En este punto, y siguiendo la idea de la corresponsabilidad, aparece un llamado a la problematización del orden sociohistórico actual, que instala con naturalidad dichas proyecciones. Advertimos aquí el profundo debate sobre el sentido de la justicia juvenil en su integralidad, y no reducido a su aspecto normativo. La adecuación de las políticas públicas para este sector poblacional adquiere aquí sentido y muestra la necesidad de recursos materiales concretos para sostener los procesos de reinserción social o, más específicamente, como enuncia la Convención Internacional de los Derechos de Niños, Niñas y Adolescentes, de reintegración social.

\section{Conclusiones}

Investigar en las ciencias sociales es un desafío enorme, y el Trabajo Social como disciplina viene recorriendo un camino arduo para poner en valor su rico y vasto potencial. La transformación de los problemas empíricos en problemas de investigación no es tarea sencilla, y el involucramiento en la construcción desde la lógica investigativa requiere de espacios institucionales que contribuyan a dichos procesos. En este sentido, el CIeCITS nos proporciona el ámbito de producción, capacitación y desarrollo de la tarea investigativa. Por ello saludamos la iniciativa de su creación y abonamos para su potenciación. La sanción de la Ley Federal de Trabajo Social (2014) incluye la investigación dentro de nuestras incumbencias profesionales, por tanto, se constituye en otro de los dispositivos habilitantes y desafiantes para rediscutir nuestro rol en las instituciones en las cuales nos encontramos insertas profesionalmente. Ambos dispositivos posibilitan el desarrollo de iniciativas como la que nos encontramos desplegando, que propician el diálogo entre los procesos de intervención y los procesos de investigación en los espacios institucionales concretos, en nuestro caso la administración de justicia. Diálogo no solo en términos formales, sino en la valorización del enfoque sociojurídico, una perspectiva que enriquece y en la que se nutren mutuamente el saber jurídico y el saber social. En este sentido, la adopción de dicho enfoque se muestra como un posicionamiento válido y como punto de encuentro entre perspectivas y áreas científicas diversas, no solo en relaciones de intercambio, sino, como bien lo plantea Calvo-García (2010), en el establecimiento de relaciones de producción. 
De Bella

Llevar adelante la tarea de investigar y sostener un proyecto de investigación requiere asumir el rol de dirección, en mi caso en particular, desde una postura democrática y participativa. Así, la construcción de la labor de investigadoras se entiende como un proceso en el que lo explícito e implícito se juegan todo el tiempo, y su abordaje se realiza desde la idea de la grupalidad, la capacitación y la puesta en práctica del proyecto, en una dinámica donde predomina la operatividad del equipo de investigación. La tarea de dirección implica asumir las contradicciones y obstáculos que surgen en el proceso de investigar. Particularmente, en el contexto de pandemia, haber podido continuar con la planificación si bien esta se vio modificada por la situación sanitaria y las medidas institucionales dispuestas- nos ha permitido desarrollar el proyecto, rectificar lo necesario y valorar la profundidad del encuentro con las y los entrevistados. Nos encontramos ahora en la fase de retomar las entrevistas, durante las que hemos podido reseñar algunos hallazgos, los cuales ratifican la pertinencia del proyecto y nos animan a desarrollarlo en su totalidad, produciendo en nosotras como equipo de investigación un plus de satisfacción en nuestro quehacer profesional cotidiano.

En el presente artículo hemos querido mostrar la trastienda de un proceso de investigación en curso, así como nuestras dudas y temores, que se van resolviendo colectivamente. Aquí la capacitación -mediante lecturas compartidas, asistencia a cursos, etc.- y la puesta en práctica adquieren un sentido primordial. La posibilidad de conformar el equipo de investigación con docentes y trabajadoras sociales vinculadas al ámbito judicial, como mencionáramos, nos sitúa en el inicio y desarrollo de una experiencia de producción de conocimiento e investigación inédita. Asimismo, dicha posibilidad permite realizar un aporte a las políticas públicas en materia de justicia, desde un TS integrador, intersectorial e interdisciplinario. La perspectiva socio jurídica nos invita al desafío de lo transdisciplinar para ampliar las perspectivas del conocimiento, validando la pertinencia del enfoque cualitativo para recuperar las voces de las y los jóvenes-adolescentes y revisar las prácticas institucionales que vinculan al Trabajo Social con lo jurídico en un determinado orden sociohistórico. Deseamos volver a encontrarnos en otra oportunidad, con el proyecto ya concluido, para continuar debatiendo y profundizando en esta hermosa tarea que es la investigación.

\section{Referencias bibliográficas}

Achilli, E. L. (2015). Investigar en antropología social. Los desafios de transmitir un oficio. Rosario, Argentina: Laborde.

Andrade-Castro, P. de. (julio, 2005). Jovens em condição de marginalidade social e ato infracional infanto juvenil no estado do Rio de Janeiro. En I Congreso Latinoamericano de Antropología. Universidad Nacional de Rosario. Facultad de Humanidades y Artes, Rosario, Argentina. 
De Bella

Bella, K. de. (2016). Justicia juvenil y trabajo social. La incidencia de las intervenciones profesionales del trabajo social en las trayectorias de los jóvenes en situación de conflicto con la ley (Tesis doctoral). Universidad Nacional de Rosario. Facultad de Ciencia Política y Relaciones Internacionales. Rosario, Argentina. Recuperado de https://rephip.unr.edu.ar/handle/2133/7895.

Beloff, M. (2001). Algunas confusiones en torno a las consecuencias jurídicas de la conducta transgresora de la ley penal en los nuevos sistemas de justicia latinoamericanos Numero 3. En Fondo de las Naciones Unidas para la Infancia-UNICEF, Justicia y derechos del niño, (pp. 9-36). Buenos Aires, Argentina: UNICEF.

Beloff, M., Freedman, D., y Terragni, M. (2013). Reseña del informe “Justicia juvenil y derechos humanos en las Américas". Revista Derechos Humanos, 2(2), 205-230. Recuperado de http://www.saij.gob.ar/doctrina/dacf130283-beloffresena_informe_justicia_juvenil.htm.

Bertaux, D. (1999). El enfoque biográfico: su validez metodológica, sus potencialidades. Acta Proposiciones, (29), 1-23.

Calvo-García, M. (2010). ¿Cabe el enfoque socio-jurídico en la teoría del derecho? Anales de la Cátedra Francisco Suárez, 44, 371-394. doi: 10.30827/acfs.v44i0.512.

Castrillón-Valderrutén, M. ${ }^{\text {a }}$ C. (2005). "Menores", cidadãos ou sujeitos de direito "tutelados"? Reflexões sobre as politicas e programas sociais para a infância e a adolescência no Brasil e na Colômbia (Tesis doctoral). Universidade de Brasília (UnB), Estudos Comparados sobre as Américas (Ceppac). Brasilia, Brasil.

Cillero-Bruñol, M. (1998). El interés superior del niño en el marco de la Convención de los Derechos del Niño. En E. García-Méndez y M. Beloff (Comps.), Infancia, ley y democracia: una cuestión de justicia. Buenos Aires, Argentina: Temis.

Congreso de la Nación Argentina. (22 de diciembre de 2014). Ley Federal del Trabajo Social [Ley 27.072 de 2014]. BO: 33.035.

Congreso de la Nación Argentina. (28 de septiembre de 2005). Ley de Protección Integral de los Derechos de Las Niñas, Niños y Adolescentes [Ley 26.061 de 2005]. BO: 30767.

D’Antonio, D. H. (2004). El menor ante el delito: incapacidad penal del menor. Régimen jurídico, prevención y tratamiento. Buenos Aires, Argentina: Astrea.

Daroqui, A., López, A. L., y Cipriano-García, R. F. (Coords.) (2012). Sujeto de castigos. Hacia una sociología de la penalidad juvenil. Rosario, Argentina: Homo Sapiens.

Dassi, T., \& Reis, M. J. (noviembre, 2008). "Mundo à parte": As relaçoes entre adolescentes e funcionários em um centro de internamento provisório. En V Jornadas de Investigación en Antropología Social. Universidad de Buenos Aires, Buenos Aires, Argentina.

Degano, J. (2004). Minoridad y Rehabilitación. Estudio crítico de las posibilidades rehabilitatorias de las prácticas judiciales actuales. (Tesis doctoral). Universidad Nacional de Rosario. Facultad de Psicología. Rosario, Argentina. Recuperado de https:/ / rephip.unr.edu.ar/xmlui/handle/2133/10912. 
De Bella

Díaz de León, L., y González-Plancencia, L. (2004). La justicia de niños y niñas en conflicto con la ley penal. Aproximación empírica a su funcionamiento. Delito y sociedad. Revista de ciencias sociales, 1(20), 29-60. doi: 10.14409/dys.v1i20.5851.

Donzelot, J. (2008). La policía de las familias. Buenos Aires, Argentina: Nueva visión.

Fernández, S. L. (2013). Protecciones debidas. Ciudadanía y gobierno de la infancia en Santa Fe (2008-2012). (Tesis doctoral). Universidad Nacional de Rosario. Facultad de Ciencia Política y Relaciones Internacionales. Rosario, Argentina. Recuperado de https:/ / rephip.unr.edu.ar/handle/2133/8734.

Firpo, S. M. (2013). La construcción social y subjetiva de la infancia y adolescencia. Buenos Aires, Argentina: Letra Viva.

García-Méndez, E. (1994). Infancia. De los derechos y de la justicia. Buenos Aires, Argentina: Ediciones del Puerto.

García-Méndez, E. (1997). Derecho de la infancia-adolescencia en América Latina: de la situación irregular a la protección integral. Bogotá, Colombia: Forum Pacis.

García-Méndez, E. (Comp.). (2008). Protección Integral de Derechos de Niñas, Niños y Adolescentes. Análisis de la Ley 26.061. Buenos Aires, Argentina: Ediciones del Puerto.

Graziano, F. (2017). Pequeños juicios. Moralidades y derechos en la administración judicial para "menores" en la ciudad de Buenos Aires. Buenos Aires, Argentina: Antropofagia.

Grinberg, J. (2004). Características y funcionamiento del entramado burocrático de protección y atención de la infancia en la Ciudad de Buenos Aires. Zonas grises en torno a las intervenciones con chicos. (Tesis de pregrado). Universidad de Buenos Aires. Facultad de Filosofia y Letras. Buenos Aires, Argentina. Recuperado de http:/ / repositorio.filo.uba.ar/handle/filodigital/959.

Guber, R. (2018). “Volando rasantes”... etnográficamente hablando. Cuando la reflexividad de los sujetos sociales irrumpe en la reflexividad metodológica y narrativa del investigador. En J. I. Piovani y L. Muñiz-Terra (Coords.), ¿Condenados a la reflexividad? Apuntes para repensar el proceso de investigación social (pp. 52-72). Buenos Aires, Argentina: CLACSO/Biblos.

Guemureman, S. (2011). La cartografía moral de las prácticas judiciales en los tribunales de menores: los tribunales orales en la ciudad de Buenos Aires. Buenos Aires, Argentina: Del Puerto.

Guemureman, S. (2020). Punitivismo judicial y dictado de sentencias por parte de los jueces de menores en Argentina. Revista Nuestra América, 8(15), 101-115. Recuperado de http:/ / observatoriojovenesiigg.sociales.uba.ar/wpcontent/uploads/sites/113/2020/06/205-470-1-PB.pdf.

Legislatura de la Provincia de Santa Fe. (19 de Marzo de 2009). Modifica Leyes 0160/1987, Ley 11452/1996 Promoción y Protección Integral de los Derechos de las Niñas, Niños y Adolescentes [Ley 12.967 de 2009]. BO: 22 de Abril de 2009.

Legislatura de la Provincia de Santa Fe. (20 de diciembre de 1996). Código Procesal de Menores de la Provincia de Santa Fe [Ley 11.452 de 1996]. BO: 20842. 
De Bella

Leo, G. de. (1985). La justicia de menores: La delincuencia juvenil y sus instituciones. Barcelona, España: Teide.

Lins-Ribeiro, G. (1989). Descotidianizar. Extrañamiento y conciencia práctica, un ensayo sobre la perspectiva antropológica. Cuadernos de Antropología Social, (3), 65-69. doi: 10.34096/cas.i3.4852.

Marcón, O. (2017). Justicia Juvenil: de las Cicatrices de la Conquista a la imaginación no Punitiva (en Perspectivas Postcoloniales). Buenos Aires, Argentina: Espacio Editorial.

Marcón, O. A. (2011). Jóvenes en situación de conflicto con la ley penal: ¿cómo relatan sus historias? Análisis y prospectivas desde la Justicia Juvenil Restaurativa. Buenos Aires, Argentina: Teseo.

Marcón, O. A. (2013). La responsabilización penal juvenil como nuevo relato cultural. ¿Del "amor por los niños" al "odio hacia los menores"? Buenos Aires, Argentina: Espacio Editorial.

Medina, F. (2021). ¿Cuánto tiempo me llevará salir de todo esto? una aproximación etnográfica a la aplicación de "medidas alternativas" sobre jóvenes en Santiago del Estero. Cuadernos de Antropología Social, (53), 137-152. doi: 10.34096/cas.i53.8480.

Miguez, D. (2008). Delito y cultura. Los códigos de la ilegalidad en la juventud marginal urbana. Buenos Aires, Argentina: Biblos.

Miguez, D., y González, Á. (2003). El Estado como palimpsesto. Control social, anomia y particularismo en el sistema penal de menores de la provincia de Buenos Aires, una aproximación etnográfica. En A. Isla y D. Miguez (Comps.), Heridas urbanas. Violencia delictiva y transformaciones sociales en los noventa (pp. 157-208). Buenos Aires, Argentina: Editorial de las Ciencias/FLACSO.

Muñiz-Terra, L., Frassa, J., y Bidauri, M. P. (2018). Hacia un encuentro de reflexividades: la entrevista biográfica como interludio del proceso de investigación social. En J. I. Piovani y L. Muñiz-Terra (Coords.), ¿Condenados a la reflexividad? Apuntes para repensar el proceso de investigación social (pp. 120-146). Buenos Aires, Argentina: CLACSO/Biblos.

Muzzopappa, E., y Villalta. C (2011). Los documentos como campo. Reflexiones teóricometodológicas sobre un enfoque etnográfico de archivos y documentos estatales. Revista Colombiana de Antropología, 47(1), 13-42.

Nebra, M. J. (2015). “Los pibes chorros” Jóvenes en situación de vulnerabilidad penal y construcción de identidad (es): políticas sociales y prácticas culturales de y para los jóvenes en conflicto con la ley. Horizontes Sociológicos, 3(6), 106-118. Recuperado de http://www.pensamientopenal.com.ar/system/files/2016/01/miscelaneas42857.pdf.

Platt, A. (2006[1969]). Los "salvadores del niño" o la invención de la delincuencia. Ciudad de México, México: Siglo Veintiuno.

Poder Ejecutivo Nacional de Argentina. (20 de marzo de 2020). Aislamiento Social Preventivo y Obligatorio [Decreto 297/2020].

Polola, D. (2004). De niños y de menores. Los profesionales y el complejo mapa institucional de la "minoridad" en la ciudad de Rosario (Tesis de pregrado). Universidad Nacional del 
De Bella

Rosario. Facultad de Humanidades y Artes. Escuela de Antropología. Rosario, Argentina.

Presidencia de la Nación Argentina. (28 de agosto de 1980). Régimen Penal de la Minoridad [Ley 22.278 de 1980]. BO: 9/5/83.

Roovers, A. (2003). Los jóvenes tutelados: un "elenco estable". En A. Isla y D. Miguez (Comps.), Heridas urbanas. Violencia delictiva y transformaciones sociales en los noventa (pp. 117-155). Buenos Aires, Argentina: Editorial de las Ciencias/FLACSO.

Ruiz-Bry, M. E. (2011). Angelitos negros... que también se van al cielo... Infancia y adolescencia encarcelada. Estudio de caso con menores detenidos en instituciones correccionales y comisarías. Algunas reflexiones sobre los derechos humanos. Rosario, Argentina: UNR Editora.

Taylor, S. J., y Bodgan, R. (1998). Introducción a los métodos cualitativos de investigación. La búsqueda de significados. Buenos Aires, Argentina: Paidós.

Tedesco, G. M. (2007). Compartiendo "caretas" y dando piñas. Construcción de vínculos y diversidad entre jóvenes en institutos correccionales. Recuperado de http://legacy.flacso.org.ar/uploaded_files/Publicaciones/Antropologia_TedescoP AVCordoba.Nro.4.pdf.

Velurtas, M. C. (2017). Intervenciones y prácticas, el trabajo social en el campo de la justicia penal juvenil. Buenos Aires, Argentina: Espacio Editorial.

Villalta, C. (2004). Una filantrópica posición social: los jueces en la justicia de menores. En S. Tiscornia (Comp.), Burocracias y violencias. Estudios de antropología jurídica (pp. 281326). Buenos Aires, Argentina: Antropofagia/Facultad de Filosofía y Letras (UBA).

Villalta, C. (2013). Un campo de investigación.: las técnicas de gestión y los dispositivos jurídico-burocráticos destinados a la infancia pobre en la Argentina. Civitas, Revista de Ciencias Sociales 13(2), 235-258. Recuperado de https://www.aacademica.org/carla.villalta/53.pdf.

Villalta, C. (2021). Antropología de las intervenciones estatales sobre la infancia, la adolescencia y la familia. Etnografiando prácticas cotidianas, construyendo perspectivas analíticas. Cuadernos De antropología Social, (53), 21-37. doi: 10.34096/cas.i53.10169. 


\section{OTROS ARTÍCULOS DE PROSPECTIVA No. 33}

\section{EDITORIAL}

El Paro Nacional en Colombia 2021: explosión social entre dinámicas estructurales y de coyuntura. Relevancia de la acción política y del diálogo en su desarrollo y transformación Adolfo Adrián Álvarez-Rodríguez

\section{ARTÍCULOS}

Organizaciones sociales de pobladores y prácticas de resistencia en contexto de pandemia COVID-19 en Chile

Patricia Castañeda-Meneses

Entre el aislamiento y las brechas digitales: sistematización de experiencia de acompañamiento socioemocional en personas mayores de Temuco, Chile, en tiempos de COVID-19

G. Bernarda Aedo-Neira

Percepción de apoyo social y calidad de vida: la visión de personas mayores chilenas en el contexto de pandemia durante el 2020

Claudia Elena Quiroga-Sanzana

Gabriela Rocío Parra-Monje

Camila Julia Moyano-Sepúlveda

Marco Alejandro Díaz-Bravo

A propósito del qué, cómo y para qué investigar en el campo de la justicia juvenil: La trastienda de un proceso de investigación en Rosario, Argentina Karina De Bella

Proyección social: reflexión sobre una experiencia virtual con adolescentes en época de pandemia en Sucre y Bolivar, Colombia

Claudia Yaneth Martínez-Mina
La intervención social mediante proyectos comunitarios en modalidad virtual en Mérida, México. Una perspectiva desde el Trabajo Social Claudia Isabel Tzec-Puch Amairani Aracelly Ceh-Alvarado Yanet Guadalupe González-Canul

Resignificación de los vínculos Sociales durante la pandemia de COVID-19: narrativas de personas que convergen en Conjuntos residenciales en Bogotá, Colombia

Andrea Marcela Reyes-García

Laura Daniela Molina-Ortiz

La paradoja de la familia doblemente recluida: clínica de una pandemia en Colombia

Norman Darío Moreno-Carmona

Juan José Cleves-Valencia

Ciencias sociales, mundo y pandemia: por un futuro posible

José Miguel Segura-Gutiérrez

Lina Paola Vásquez-Ávila

¿Qué es Trabajo Social? Los primeros vínculos de los estudiantes con la profesión: experiencias de Argentina, Brasil, Paraguay y Uruguay

Silvia Orieta Rivero-Rodríguez

Leonel Del Prado

Nidia Graciela Battilana-Amarilla

Rosilaine Coradini-Guilherme

Intervención profesional de trabajadores sociales de la universidad del Quindio, Colombia. Asuntos epistemológicos, metodológicos y ético-políticos

Ana María Gil-Ríos 
Reflexiones sobre la intervención social en lo rural: experiencias en el Magdalena Medio, Colombia

Claudia Milena Quijano-Mejía

Johana Linares-García

Construcción de rutas de atención integral a la convivencia escolar en Cali, Colombia: sistematización de la experiencia

Leidy Johana Prado-Montaño

Luz Helena López-Rodríguez

Alejandra Gutiérrez-Cárdenas

Experiencia del proceso de intervención familiar de los actores participantes en la Escuela para Familias del ICBF-Jamundí, Colombia Katherin Viviana Silva-Minotta

María José Forero-Izquierdo

Jimena del Pilar Jaramillo-Jaramillo

\section{RESEÑAS DE LIBROS}

Alerta global: políticas, movimientos sociales y futuros en disputa en tiempos de pandemia Jairo Crispín

El neoliberalismo como teología política. Habermas, Foucault, Dardot, Laval y la historia del capitalismo contemporáneo

José Francisco Desentis-Torres

La Reconceptualización del Trabajo Social en Colombia: Análisis histórico-crítico de las décadas de 1960-1970

Juan Pablo Sierra-Tapiro

ARTISTA INVITADO

Juan Camilo González

Prospectiva

\section{PROSPECTIVA}

Revista de Trabajo Social e Intervención Social

No. 33 • ene.-jun. 2022

e-ISSN: 2389-993X • Universidad del Valle 